\title{
Breast metastasis from rectal carcinoma: A case report and review of the literature
}

\author{
Dan-Dan Wang ${ }^{1}$, Su-Jin Yang ${ }^{1}$, Wei-Xian Chen ${ }^{* 2}$
}

1. Department of Breast Surgery, The First Affiliated Hospital of Nanjing Medical University, Nanjing 210029, Jiangsu Province, China

2. Department of Breast Surgery, The Affiliated Changzhou No. 2 People's Hospital of Nanjing Medical University, Changzhou 213000, Jiangsu

Province, China

Correspondence: Wei-Xian Chen (chenweixian@njmu.edu.cn)

\section{Background}

Metastasis from extramammary primary tumor to breast is extremely rare.

Case Summary

A 59-year-old woman with 1-year history of rectal cancer presented with asymptomatic breast mass. At 16 months after the diagnosis of rectal mucinous adenocarcinoma, a breast mass was confirmed by ultrasonography and identified by pathology and immunohistochemistry as a metastasis from the rectal cancer. Treatments included chemotherapy (6 cycles: $300 \mathrm{mg}$ irinotecan on day 1 , $4.5 \mathrm{mg}$ raltitrexed on day 2, $450 \mathrm{mg}$ bevacizumab on day 3), radiotherapy, and surgical resection. Two years of follow-up examinations (6-months intervals) showed no evidence of recurrence or novel distant metastasis.

Conclusion

Breast metastasis from rectal carcinoma is a rare secondary malignancy. Final diagnosis can be established by histopathology and immunohistochemistry.

Key words: Breast cancer; Rectal carcinoma; Metastasis; Case report; Secondary malignancy; Review

\section{Case presentation}

We report a case of breast metastasis from rectal carcinoma in a middle-age woman who had been diagnosed and treated for the primary rectal cancer 16 months prior to the noticeable development of a secondary breast tumor.

A 59-year-old female visited our hospital in August 2017, complaining of breast mass. Upon presentation at our hospital, the palpable left-breast mass was subjected to imaging examinations. Ultrasonography revealed the mass to be an irregular, ill-defined and hypoechoic tumor (BI-RADS 5), measuring $19 \mathrm{~mm} \times 15 \mathrm{~mm}$ (Figure $1 \mathrm{~A})$. X-ray showed the mass to be $16.7 \mathrm{~mm} \times 19.4 \mathrm{~mm}$ (Figure $1 \mathrm{~B}$ ). MRI displayed a single enhancing lesion in left breast (Figure 1D and E). A tumor sample was obtained by rough needle puncture, and subsequent immunohistochemistry (IHC) staining indicated invasive breast cancer with signet ring cell carcinoma (Figure 1C). Immunotyping showed negativity for breast cancer markers like estrogen receptor (ER), progesterone receptor (PR) and human epidermal growth factor receptor-2 (Her-2) but positivity for rectal cancer markers such as cytokeratin (CK)7, CK20, villin, CDH17 and CDX-2; Ki-67 detection showed a $20 \%$ positive rate.

Past medical history showed that the patient had a 2-months history of recurrent diarrhea and anal dilatation in April of 2016. Routine blood investigations showed results in the normal ranges. Abdominal contrast-enhanced computed tomography (CT) showed a mass in the rectum that was accompanied by hyperplastic lymph nodes (Figure 2A). Pathology assessment of a biopsy sample obtained via colonoscopy provided the diagnosis of mucous adenocarcinoma and signet ring cell carcinoma (images unavailable). Further evaluation by magnetic resonance imaging revealed the tumor to be in the rectum, $\sim 5.1 \mathrm{~cm}$ diameter in size and $\sim 5.5 \mathrm{~cm}$ from the anus and encircling the intestinal cavity and a swollen lymph nodes; the findings prompted suspicion of metastasis (Figure 2B). Following multidisciplinary care team discussion, the patient was started on a neoadjuvant radiotherapy and chemotherapy. From June 30, 2016 to August 4, 2016, the patient received $50 \mathrm{~Gy} / 25 \mathrm{~F}$ radiation (2 Gy each visit, Monday to Friday for 5 weeks) and 7-days rounds of $1500 \mathrm{mg} \mathrm{Xeloda}^{\circledR}$ (Roche, Shanghai, China) twice daily from day 1 to day 5 with rest on day 6 and day 7. On August 30, 2016, the neoadjuvant chemotherapy was changed to a 14-days regimen of $400 \mathrm{mg}$ oxaliplatin (Hengrui, Jiangsu, China) on day 1 and $1500 \mathrm{mg}$ Xeloda ${ }^{\circledR}$ twice daily from day 1 to day 14 (Figure 2C). On October 14,2016 , the patient underwent abdominoperineal resection (Miles' procedure) and the subsequent pathology assessment provided the diagnosis of mucinous adenocarcinoma (pT3N1M0; Figure 2F). The chemotherapeutic regimen was changed to an 8-cycles program with once every 14 days of $200 \mathrm{mg}$ oxaliplatin on day 1 and $1500 \mathrm{mg}$ Xeloda twice daily from day 1 to day 14, and the last time of chemotherapy is March of 2017. The cancer appeared to be resolved (Figure $2 \mathrm{D}$ and $\mathrm{E}$ ).

Taking into account the breast metastasis originating from a primary rectal cancer, the following chemotherapy regimen was ordered: 6-round, 3-days course of $300 \mathrm{mg}$ irinotecan (Qilu Pharmaceutical, Hainan, China) on day 1, $4.5 \mathrm{mg}$ raltitrexed (Zhengda Tianqin Pharmaceutical Co., Nanjing, China) on day 2, and $450 \mathrm{mg}$ bevacizumab (Roche, Shanghai, China) on day 3. On May 10, 2018, the patient underwent enlarged resection of the left-breast tumor (pathologyverified clear margins). The pathology assessment showed mucinous adenocarcinoma and IHC evaluation showed 
A

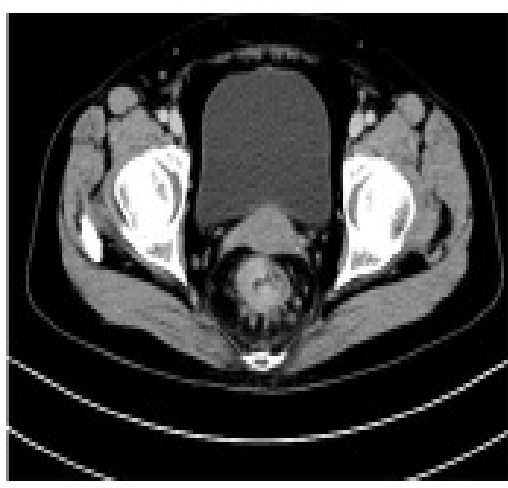

D

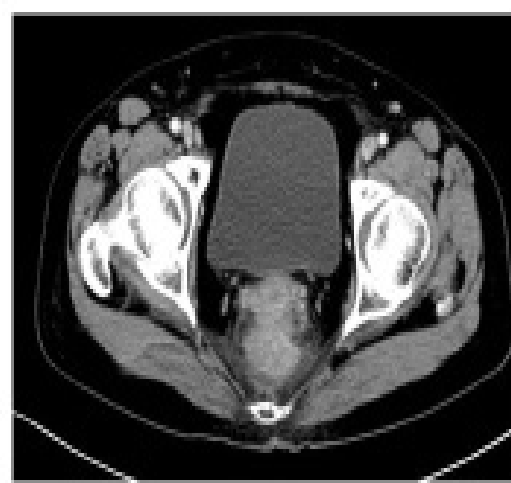

B

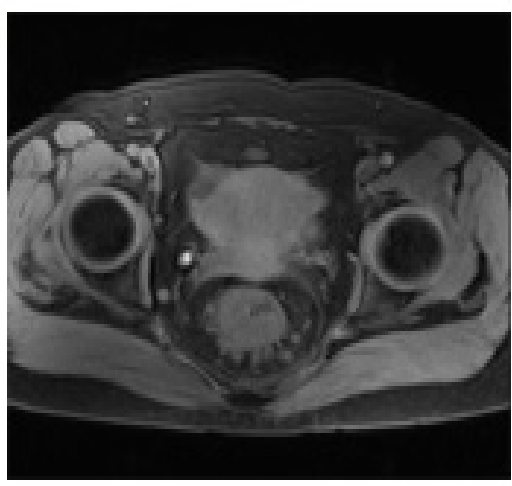

E

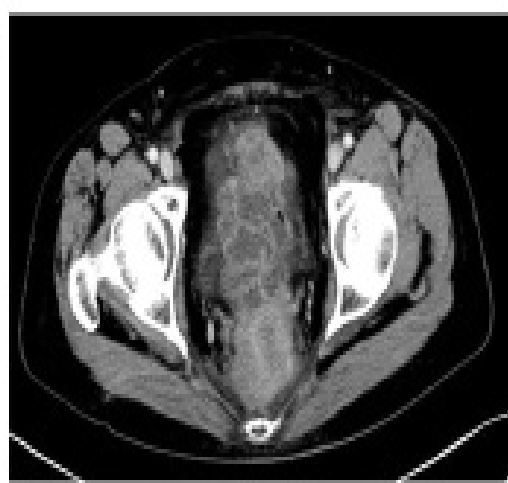

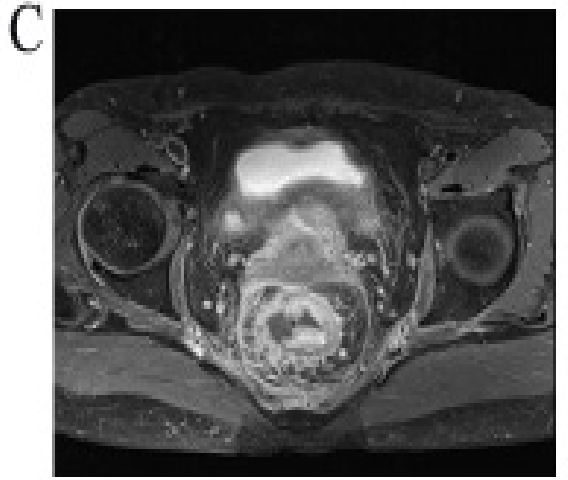

F

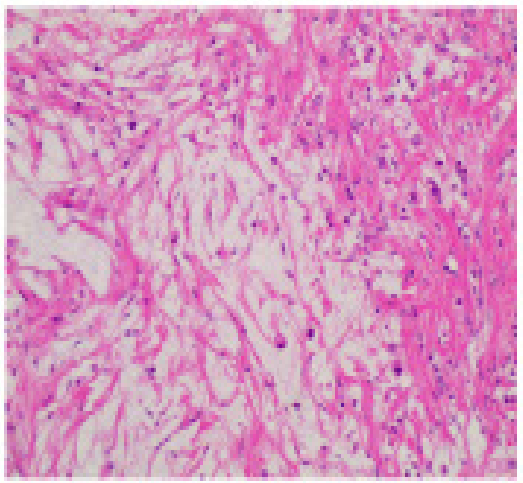

Figure 1 Imaging findings and pathological characteristics of the patient's breast metastasis. A: Ultrasonography revealed a mass in the left breast; B: X-ray of the left breast; C: Pathology (hematoxylin and eosin) of the rough needle puncture of the tumor; D: Axial magnetic resonance imaging (MRI) of the breasts; E: Coronal MRI of the left breast; F: Pathology (hematoxylin and eosin) of the resected tumor.
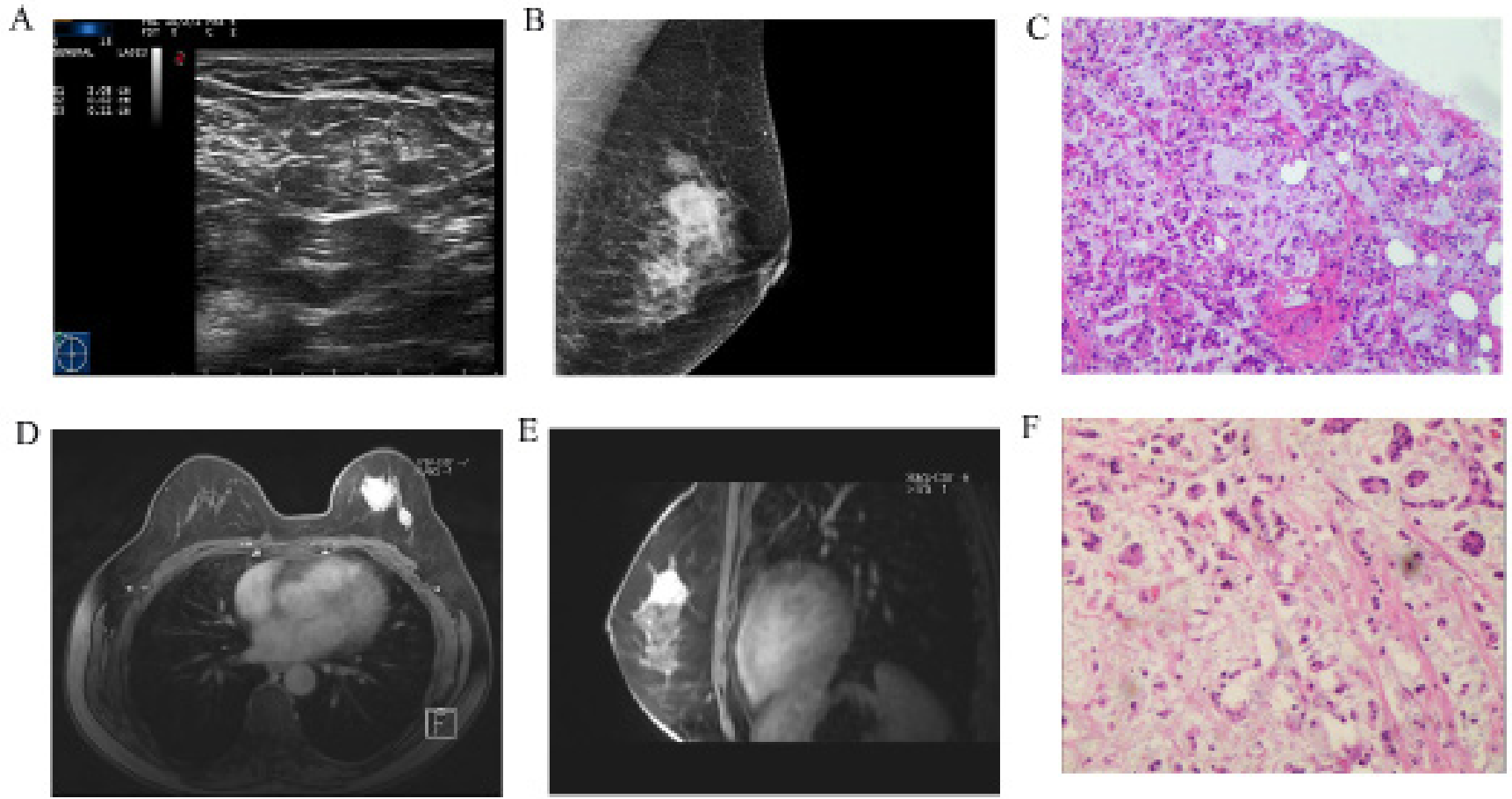

Figure 2 Imaging findings and pathological characteristics of the patient's previous rectal carcinoma. A: Computed tomography (CT) showed a mass in the rectum (thick red arrow), accompanied by hyperplastic lymph nodes (thin red arrow); B: Magnetic resonance imaging (MRI) also showed the rectal cancer (red arrows); C: CT image made after the adjuvant chemotherapy and radiotherapy, showing regression of the tumor (red arrow); D and E: CT and MRI images, respectively, made after the Miles' surgical procedure; F: Pathology (hematoxylin and eosin) showed mucinous adenocarcinoma.

diarrhea and mucous adenocarcinoma and anal dilatation signet ring cell carcinoma chemotherapy and radiotherapy
Miles chemotherapy $\quad \begin{gathered}\text { breast cancer } \\ \text { from rectal cancer }\end{gathered}$ chemotherapy breast surgery

Apr.2016 Jun.2016 Jun.2016--Aug.2016 $\quad$ Oct.2016 $\quad$ Nov.2016--Mar.2017 $\quad$ Aug.2017 Sep.2017--Jan.2018 May.2018

Figure 3 Timeline of multimodal treatments. 
positivity for CK20, CDH17, SATB2, CDX-2 and Ki-67 but negativity for ER, PR, and Her-2 (Figure 1F and Figure 3). After breast surgery, the patient was admitted to the Oncology Department for postoperative chemotherapy. Follow-up examinations have been performed every 6 months, with no evidence of recurrence or novel distant metastasis up to the most recent 2-year follow-up.

\section{Discussion}

Colorectal cancer is the third most common cancer in humans and the second leading cause of mortality due to distant metastasis ${ }^{[1]}$. The most common areas of metastasis from rectal cancer are liver, lung and brain ${ }^{[1]}$. Breast metastasis from rectal cancer cases are extremely rare in clinical practice, with only 10 such patients having been described in the literature ${ }^{[2-7]}$. We reason that secondary breast metastasis may be a special type of rectal cancer, with a metastatic pathway involving blood or an axillary vein. The first step in diagnosis is to definitively identify whether the breast mass is a primary tumor or metastasis; this can be accomplished by combining the results of pathology and IHC. ER, PR and Her-2 are biomarkers for breast cancer, and CK20, CDH17, SATB2 and CDX-2 are biomarkers for rectal cancer ${ }^{[8,9]}$. Additionally, some other suspected immunohistochemical makers depended on what cancer is suspected. Moreover, it is important to identify any case of "triple-negative" (ER, PR, Her-2) breast cancer according to the previous malignancy and history, as such requires specialized treatment.

At present, the pathogenic mechanism of rectal cancer metastasizing to the breast remains unclear. Rectal canceroriginating metastatic disease in the breast, itself, is a marker for disseminated metastatic spread and indicates a poor prognosis. For these patients, we suggest performing a general imaging examination (e.g., positron emission tomographyCT) to evaluate the general state and monitor the response to treatment. Recently, gene sequencing for cancers has become a hot topic of investigation, with some studies showing benefit to the patients, such as effectiveness in detecting curative effects of targeted medicine ${ }^{[10]}$. Furthermore, radiofrequency ablation (commonly referred to as RFA) is an established procedure for treatment of various advanced cancers $^{[11,12]}$. A systematic review showed RFA to be feasible and safe with only minor related complications in breast cancer patients ${ }^{[13]}$. For our patient, if the breast metastasis recurs, we may consider RFA as one of the potent treatment. Herein, we report a rare case of breast metastasis from rectal carcinoma (Figure 3). The diagnosis was established by good history of precious malignancy, histopathology and IHC findings. Reporting of this case may help in the development of personalized medicine, targeted therapy, and immunotherapy approaches that will provide effective strategies to better manage future patients with this rare disease entity.

\section{References}

1. Siegel RL, Miller KD, Jemal A. Cancer statistics, 2018. CA Cancer J Clin. 2018;68(1):7-30.

2. Lal RL, Joffe JK. Rectal carcinoma metastatic to the breast. Clin Oncol (R Coll Radiol). 1999;11(6):422-3.

3. Li J, Fang Y, Li A, et al. Breast metastases from rectal carcinoma. Chin Med J (Engl). 2011;124(8):1267-9.

4. Mihai R, Christie-Brown J, Bristol J. Breast metastases from colorectal carcinoma. Breast. 2004;13(2):155-8.

5. Arıbas BK, Onursever A, Kiziltepe T, et al. Breast Metastasis from Rectal Adenocarcinoma: A Case Report with US and CT Findings. Imaging in Medicine. 2016;8(3):89-92.

6. Singh T, Premalatha CS, Satheesh CT, et al. Rectal carcinoma metastasizing to the breast: a case report and review of literature. J Cancer Res Ther. 2009;5(4):321-3.

7. Sanchez LD, Chelliah T, Meisher I, et al. Rare case of breast tumor secondary to rectal adenocarcinoma. South Med J. 2008;101(10):10624.

8. Ryu A, Ashimura JI, Nakayama T, et al. Reliability of Estrogen Receptor and Human Epidermal Growth Factor Receptor 2 Expression on Breast Cancer Cells Stored in Cellprep ${ }^{\circledR}$ Vials. Acta Cytol. 2018;62(5-6):360-370.

9. Tsujimura K, Teruya T, Kiyuna M, et al. Colonic metastasis from breast carcinoma: a case report. World J Surg Oncol. 2017;15(1):124.

10. Huang JL, Kizy S, Marmor S, et al. Tumor grade and progesterone receptor status predict 21 -gene recurrence score in early stage invasive breast carcinoma. Breast Cancer Res Treat. 2018;172(3):671-677.

11. Hua YQ, Wang P, Zhu XY, et al. Radiofrequency ablation for hepatic oligometastatic pancreatic cancer: An analysis of safety and efficacy. Pancreatology. 2017;17(6):967-973.

12. Bale R, Richter M, Dünser M, et al. Stereotactic Radiofrequency Ablation for Breast Cancer Liver Metastases. J Vasc Interv Radiol. 2018;29(2):262-267.

13. Ito $\mathrm{T}$, Oura $\mathrm{S}$, Nagamine $\mathrm{S}$, et al. Radiofrequency Ablation of Breast Cancer: A Retrospective Study. Clin Breast Cancer. 2018;18(4):e495-e500. 